\title{
Die Alternative für Deutschland
}

\author{
Eine gefährliche Kraft im gesellschaftlichen Wandel
}

Die Zeit des Ignorierens ist längst vorbei. Die "Alternative für Deutschland" (AfD) hat sich als feste Größe in den deutschen Parlamenten sowie im Europaparlament verankert. Doch was ist sie? Ist sie eine Partei, die an die CDU vor Heiner Geißler und Angela Merkel anknüpfen will oder ist sie auf dem Weg, eine systemsprengende Kraft zu werden, die das politische System grundsätzlich herausfordert? Oder ist sie vielleicht etwas Drittes? Offensichtlich ist, dass sie Ausdruck einer sich verändernden politischen Konstellation ist, in der sich Politikinteresse, Protestverhalten und Parteien neu ordnen.

I m Zentrum der wissenschaftlichen Debatte stehen Überlegungen, die die AfD als Phänomen der Modernisierungsverlierer_innen oder als Ausdruck einer neuen gesellschaftlichen Spaltungslinie identifiziert, die auf den tiefgreifenden Konflikt zwischen Kosmopolitismus contra Kommunitarismus zurückgehe. Im Hinblick auf die AfD-Anhängerschaft lässt sich der Zulauf zu dieser Partei vor allem so erklären: Erstens als Folge

\footnotetext{
Wolfgang Schroeder

Politisches System der BRD - Staatlichkeit im Wandel, Universität Kassel, Kassel, Deutschland

*1960; Prof. Dr., Hochschullehrer für das „Politische System der BRD” an der Universität Kassel und Research Fellow am Wissenschaftszentrum Berlin (WZB). Forschungsschwerpunkte: Arbeitsbeziehungen, Gewerkschafts- und Verbändeforschung, Sozialstaat. wolfgang.schroeder@uni-kassel.de
}

\section{Bernhard Weßels}

Demokratie und Demokratisierung, Wissenschaftszentrum Berlin, Berlin, Deutschland *1955; Prof. Dr., Hochschullehrer an der Humboldt-Universität zu Berlin und stellv. Direktor der Abteilung „Demokratie und Demokratisierung” am Wissenschaftszentrum Berlin für Sozialforschung. Forschungsschwerpunkte: comparative political behavior, Interessenvermittlung und politische Repräsentation.

bernhard.wessels@wzb.eu

Zusammenfassung Die AfD hat es binnen kurzer Zeit geschafft, in alle deutsche Landesparlamente einzuziehen sowie als Bundestags-Novizin die größte Oppositionsfraktion zu stellen. Gleichwohl bleibt sie mit ihren inneren Dynamiken, den Wandlungs- und Transformationsprozessen sowie der heterogenen Koalition von bewegungs- und parlamentsorientierten Kräften ein rätselhaftes Phänomen. Vor diesem Hintergrund beleuchtet der Beitrag die Entwicklung der Partei, ihr programmatisches und parlamentarisches Profil sowie die Abgrenzung und Bezugnahme auf rechtsextreme Akteur_innen.

Schlüsselwörter Alternative für Deutschland, Parlamentarisierung, Rechtspopulismus einer weit verbreiteten Verunsicherung, bei der sich die negativen Folgen der wirtschaftlichen und technologischen Globalisierung sowie die Angst vor Fremden in einer diffusen Melange verbinden, die durch eine neue Politik der Emotionalisierung beantwortet wird. Zweitens, indem die Partei diese emotionalisierte Herangehensweise an die reklamierten und zum Teil auch selbst generierten Ängste dazu nutzt, um einen problematischen Rückzug auf die Interessen der deutschen Bevölkerung zu propagieren. Dabei wird die Europäische Union als Irrweg deklariert, dem die AfD ein nationalistisch aufgeladenes Verständnis der Schutzmacht des nationalen Staates gegenübergestellt.

\section{Der Erfolg der AfD - Schließung einer Lücke}

Um welche Partei es sich bei der AfD wirklich handelt, ist gleichwohl sehr umstritten. So fällt die Antwort auf diese Frage auch in Abhängigkeit vom Zeitpunkt, den Themen und der regionalen Ausprägung der AfD unterschiedlich aus. Jedenfalls mangelt es nicht an unterschiedlichen Etiketten, die der AfD angeheftet werden: Anti-Systempartei, liberal-konservative Partei der bürgerlichen Mitte, deutsch-nationale Patrioten, rechtsextrem, rechtspopulistisch, fremdenfeindlich, autoritär, Anti-Establishment, Gegner der „Lügenpresse“, Partei der Modernisierungsverlierer_innen, Partei des Zorns, ein Chamäleon, die rechte Alternative zur Union oder gar anti-autoritär. Manche dieser Etiketten beansprucht sie selbst für sich. Rückblickend könnte man sagen, die Entwicklung der AfD als rechtspopulistische Erfolgspartei begann mit einem „Trick“. Sie gründete sich nämlich zunächst als konservativ-liberale Partei, in deren Zentrum euroskeptische Positionen standen. Zugleich bezog sie sich positiv auf die nationale Souveränität, die es aus Sicht der AfD zu fördern gelte. Damit war für den 


\section{Durchblick: Rechtsnationaler Autoritarismus und Soziale Arbeit}

Eintritt einer neuen Partei in den Parteienwettbewerb die Hemmschwelle viel niedriger, als wenn sie von Anfang an als rechtspopulistische Partei angetreten wäre. Dass Derartiges erfolgreich gelingt, setzt eine Lücke im politischen Angebot voraus, für die es eine Nachfrage gibt. Die Angebotslücke im deutschen Parteiensystem waren europakritische Positionen, die mit der Finanzund Eurokrise auch eine deutliche Nachfrage in der Bevölkerung fanden. Daneben ist aber auch ein Protest gegen den gesellschaftlichen und ökonomischen Status quo zu berücksichtigen (beispielsweise von Anhänger_ innen der Sarrazin-Debatten). Entscheidend für den Erfolg waren schließlich Übertritte zur AfD von Politiker_innen der etablierten Parteien (Decker 2016, S. 14). Ein Effekt, den die AfD erzielte, ist vermutlich, dass sie die längst gespaltene deutsche Gesellschaft zwingt, sich intensiver mit sich selbst und Widersprüchen zu befassen. In manchen Themenbereichen versucht die AfD den Eindruck zu vermitteln, sie würde Repräsentationslücken schließen und politische Alternativen anbieten. Auf jeden Fall profitiert die Partei einerseits von der neuen Ungeduld, dem neuen Zorn in Teilen der Gesellschaft. Andererseits trägt sie selbst seit einigen Jahren dazu bei, die Politikverdrossenen wieder im Kontext des politischen Systems zu mobilisieren, das Interesse an öffentlichen Diskursen anzuregen und die Wahlbeteiligung zu steigern. Zugleich wird durch ihre Präsenz und ihre Interventionen nicht nur der parlamentarische Parteienwettbewerb komplexer und das Ringen um Kompromisse schwieriger, sondern sie trägt mit ihren vermeintlichen Problemlösungsrezepten tatsächlich zu einer weiteren Spaltung der Gesellschaft bei.

Insofern ist die AfD einerseits Ausdruck gegenwärtiger, tiefer gesellschaftlicher Widersprüche und Übergänge, womit sie an damit ausgelöste Unzufriedenheit und Benachteiligung in Teilen der Bevölkerung anknüpfen kann; andererseits erscheint sie durch ihre anti-pluralistischen Versatzstücke immer wieder als systemsprengendes, demokratiegefährdendes Sammelbecken, das die verfassungsmäßigen Grundlagen des Systems mit reaktionären Inhalten (anti-egalitär, ethno-nationalistische Agenda, Anti-Gender, gegen kulturellen Pluralismus sowie universelle Menschen- und Grundrechte) und populistischen Praktiken zu torpedieren versucht. Die ihr eigene Ambivalenz besteht also darin, dass sie einerseits bestimmte Themen auf die Tagesordnung setzt, die bisher zu wenig öffentlich diskutiert oder von der etablierten Politik gemieden wurden, und andererseits mit ihren Positionen die Axt an die Wurzeln der Demokratie legt.
Das programmatische Profil und innere Dynamiken Ideologisch und programmatisch reiht sich die AfD in die Parteienfamilie des europäischen Rechtspopulismus ein, dessen Hauptmerkmal einerseits die Anti-Establishment-Orientierung ist (z. B. durch die Forderung nach „mehr direkter Demokratie“), andererseits der Anspruch, den „wahren“ Willen eines homogenen Volkes zu vertreten, verbunden mit der Abwehr von Einwanderung und Integration, die als Ursprung der meisten gesellschaftlichen Probleme gedeutet wird. Auf der wertebezogenen Agenda der AfD steht eine anti-egalitäre, minderheitskritische, ethno-nationalistische Agenda, die sich gegen Feminismus, kulturellen Pluralismus sowie universelle Menschen- und Grundrechte positioniert. Hinzu kommen starke einzelne Positionen, wie die Konzentration auf den Vorrang deutscher Interessen, gekoppelt mit einer Abwertung oder gar Distanzierung von supranationalen Institutionen und von einer Politik des Multilateralismus. Innenpolitisch werden die öffentlichen Medien als Feindbild stilisiert. Die sogenannte „Lügenpresse“ wird durch eine große Reichweite der Partei in sozialen Netzwerken umgangen, um eine unverfälschte Direktkommunikation mit der eigenen Anhängerschaft und der Öffentlichkeit zu ermöglichen.

In der Familien- und Gesellschaftspolitik vertritt die Partei stark konservative Positionen, die im Großen und Ganzen innerparteilich akzeptiert sind. Auch wenn in Bezug auf das Thema Homosexualität innere Verwerfungen und Konflikte offensichtlich sind, konnten diese Dissonanzen bislang meist intern wegmoderiert werden. Dagegen sind solche Konflikte in der Sozial- und Wirtschaftspolitik für die Partei existenzieller, was sich an den divergenten Akzenten in der Rentenpolitik zeigt, wo sich neoliberale und sozial-populistische Positionen unversöhnlich gegenüberstehen. Der AfD ist es bisher nicht gelungen, die unterschiedlichen Konzepte parteiöffentlich zu diskutieren und sich auf ein Konzept zu verständigen. Weniger stark polarisiert scheinen erste Suchbewegungen in der Arbeitsmarktpolitik zu sein, wo Forderungen nach Leistungsverbesserungen für betroffene Gruppen gestellt wurden.

Um die AfD zu verstehen, ist es wichtig, die sie tragende Koalition zwischen dem parlamentsorientierten und dem bewegungsorientierten Flügel zu analysieren. Den „Parlamentsorientierten“, die durchaus den gegebenen verfassungsrechtlichen Rahmen achten, geht es darum, sich dauerhaft als konservative Kraft jenseits der CDU zu etablieren, die perspektivisch regierungsfähig werden soll. Um dieses Ziel zu erreichen, will man die Unzufriedenen ansprechen. Die „Bewegungsorientierten“ in der AfD sind von der Idee der sozialen Bewegung geprägt. Sie wollen nicht koalitionsfähig werden, sondern 
ihr Erpressungspotenzial ausspielen, um die Richtung des Parteienwettbewerbs zu beeinflussen und haben ein eher taktisches Verhältnis zur pluralistischen Demokratie. Sie sehen sich als Sprachrohr rechter Vororganisationen und versuchen, ihre eigene Anhängerschaft auf der Straße und insbesondere im Netz zu mobilisieren. Diesem Idealtyp am nächsten kommt die thüringische Landtagsfraktion rund um ihren Fraktionsvorsitzenden Björn Höcke, der als eine der Gallionsfiguren der Neuen Rechten regelmäßig eigene AfD-Demonstrationen veranstaltet oder als Redner an ihnen teilnimmt.

Die Entwicklung der AfD ist eine Geschichte der Metamorphosen. Diese Wandlungs- und Transformationsprozesse betreffen ihre Wähler_innen, ihre Mitglieder und ihre Mandatsträger_innen. Dabei bestehen zwischen den Gruppen durchaus Ungleichzeitigkeiten und Inkompatibilitäten. So sind die neoliberalen Eurokritiker_innen der ersten Stunde mittlerweile meist von Bord gegangen (bzw. gegangen worden) oder aber sie haben sich den inhaltlichen Metamorphosen der AfD angepasst und sich sogar an deren Spitze gestellt. Bei allen kleinteiligen Verschiebungen, von permanenten Konflikten zwischen den Strömungen gekennzeichnet, sind zwei grundlegende Phasen erkennbar (Schroeder/ Weßels 2019, 23f.):

- In der ersten Phase, die durch neoliberale Eurokritiker_innen geprägt war, dominierte ein Abgrenzungskurs der Parteispitze gegenüber rechtsextremen Positionen. Das Leitbild dieser Phase bestand darin, die eigenen Positionen stark genug zu machen, um CDU und FDP in ihrem bürgerlichen Alleinvertretungsanspruch herauszufordern und sich selbst als dritte Kraft in dieser Konstellation zu etablieren.

- In der zweiten Phase, die seit etwa 2015 andauert, gibt es eine Pattposition zwischen parlamentsorientierten Kritiker_innen des Status quo und den bewegungsorientierten Aktivist_innen, die das Parteiensystem und das politische System insgesamt transformieren wollen. Mit dem Einzug in den Bundestag ist insofern ein neues Kapitel der AfD-Entwicklung aufgeschlagen worden, weil es nunmehr ein klar definiertes Kraftund Steuerungszentrum der Partei gibt, das die meisten Ressourcen hat und die öffentliche Aufmerksamkeit auf sich zieht.

\section{Abgrenzung und Bezugnahme auf rechtsextreme Akteur_innen}

Rechtsextreme Kräfte haben sich von Anfang an auf die AfD bezogen, haben versucht, sie zu unterwandern und für ihre Ziele zu instrumentalisieren. Da alle rechten Bewegungen und Parteien in Deutschland bislang an einer unzureichenden Abgrenzung zu rechtsextremen
Denk- und Handlungsmustern, Netzwerken und Personen scheitern, haben die Parlamentsorientierten durchaus daran gearbeitet, eine harte Brandmauer aufzubauen. Allerdings sind die Übergänge zwischen diffusen Unzufriedenheiten, rechtskonservativen, rechtspopulistischen und rechtsextremen Positionen fließend. Über den Umgang mit rechtsextremen Tendenzen gab es von Anfang an erbitterte Kontroversen im Bundesvorstand und den einzelnen Landesverbänden.

Mit der Radikalisierung der AfD ist auch der Verfassungsschutz auf den Plan gerufen, der im Januar 2019 die gesamte Partei zum „Prüffall“ erklärte und einzelne Teile, wie die Bundesjugendorganisation „Junge Alternative“ und den rechts stehenden „Flügel“, zum „Verdachtsfall“ erklärte. Aus Sicht des „Flügels“ sollte die „Verfassungsschutzkeule (...) die vorgeblich ,radikalen' Kräfte in der Partei schocken und die ,gemäßigten“ Kräfte locken “ (Höcke 2019). An anderer Stelle heißt es bei Björn Höcke: „Die Bedrohung mittels Beobachtung durch den Verfassungsschutz stellt (...) eine unzulässige, freiheits- und demokratiefeindliche Instrumentalisierung einer staatlichen Behörde zwecks Niederhaltung einer parteipolitischen Opposition dar. Und dennoch wittern manche Teile in der AfD die große Chance auf eine ,Bereinigung' von ungeliebten, patriotischen Kräften und begehen die Todsünde des ,Feindzeugen': Sie belasten eigene Parteikollegen und -gruppierungen mit zumeist völlig unhaltbaren ,Extremismus`-Vorwürfen.“ (Höcke 2019).

Die AfD-Führung befürchtet, dass mit den Einlassungen des Verfassungsschutzes nicht nur die Spaltung der eigenen Partei befördert werden könne, sondern auch negative Konsequenzen bei einem Teil der Wählerschaft sowie bei Spender_innen folgen könnten. Durch eigene juristische und organisatorische Gegenstrategien versucht man, die Aktivitäten des Verfassungsschutzes in die Schranken zu weisen. Ob die AfD diese neue innerparteiliche Konfliktsituation aufzulösen vermag, wird davon abhängen, ob es zu einer De-Radikalisierung oder zu einer weiteren Radikalisierung in der AfD kommt. Da die radikaleren Kräfte geschlossener, offensiver und lautstärker auftreten, wird viel davon abhängen, wie opportunistisch, politik- und leidensfähig die gemäßigteren Kräfte sind.

\section{Parlamentarisierung: Landtage, Europaparlament und Bundestag}

Die AfD ist mittlerweile in vielen kommunalen Parlamenten, in allen Landtagen, im Europaparlament und im Bundestag angekommen. Beim Blick auf die regionale Verteilung der Wahlerfolge zeigt sich, dass diese keinesfalls gleichmäßig ausfallen: Auffallend sind 


\section{Durchblick: Rechtsnationaler Autoritarismus und Soziale Arbeit}

schwache Wahlergebnisse in den norddeutschen Ländern, starke Wahlergebnisse im Süden und nahezu überragende Wahlergebnisse im Osten. Bei den Bundestagswahlen 2017 und bei den Europawahlen 2019 erreichte die sächsische AfD sogar das stärkste Ergebnis aller Parteien. Im Zuge dieser Parlamentarisierung veränderten sich auch das programmatische Profil und die Wählerschaft - letztere vor allem durch massive Zugewinne aus dem Lager der Nichtwähler_innen. Konnte die AfD in den ersten Wahlen verstärkt unzufriedene Wähler_innen der CDU, FDP, der Linkspartei und der SPD gewinnen, so spielte dieses Potenzial bei den Bundestagswahlen 2017 nur noch eine untergeordnete Rolle. Es sind nunmehr die vorherigen Nichtwähler_innen, die die Wahlerfolge der AfD maßgeblich ermöglichten.

Auffallend ist allerdings, dass es die AfD nach Frauke Petrys Austritt im Bundestag geschafft hat, die Fraktion so zusammenzuhalten, wie es ihr auf anderen parlamentarischen Ebenen bislang nicht gelungen ist. Ursächlich dafür könnte der massive äußere Druck sein, der innere Schließungsprozesse erleichtert oder sogar erzwingt. $\mathrm{Ob}$ die erreichte innere Integration auch das Ergebnis eines effizienten innerfraktionellen Managements von Konflikten ist, bedarf der empirischen Analyse. Förderlich für die innerfraktionelle Steuerung ist sicherlich, dass die Mehrheit der Abgeordneten nicht nur Bundestagsneulinge sind, sondern kaum oder gar keine parlamentarische Erfahrung mitbringen. Bislang gelang es der AfD im Bundestag, sich formell zu etablieren, ohne sich dabei gänzlich anpassen zu müssen. Die Fraktion wurde durch das Parlament bisher weder weichgeschliffen noch zeichnet sie sich durch massive innerfraktionelle Konflikte aus. Doch das sind nur oberflächliche Aufnahmen, die den Blick in die Tiefen der inneren Konflikte, Verwerfungen und Härten aussparen.

Kennzeichnend für die AfD ist ein aggressiver und konfrontativer Stil, der aus der Sicht anderer Fraktionen und Beobachter_innen in den meisten Parlamenten einen Klimawandel bewirkt. Häufig werde das Plenum von Vertreter_innen der AfD als Bühne benutzt, während die Beiträge der AfD in der Arena des Arbeitsparlaments eher wenig wahrnehmbar sind und kaum durch inhaltliche Kompetenz auffallen. An diesem Bild ändert sich auch dann nur wenig, wenn man Beobachtungen berücksichtigt, die in einzelnen Ausschüssen des Bundestages aktivere und kompetentere Vertreter_innen identifizieren als auf der Ebene der Landtagsausschüsse. Doch auch wenn die Positionen von AfD-Abgeordneten keinesfalls alle durch einen strukturierten, ideologisch klar profilierten Filter laufen, verstehen sie ihre Partei gleichwohl mehrheitlich als die einzige echte Oppositionspartei.
Im ersten Jahr ihrer Zugehörigkeit zum deutschen Bundestag (bis 01.10.2018) hat die AfD sieben Gesetzesentwürfe eingereicht und etwa 317 Kleine und drei Große Anfragen gestellt sowie drei Aktuelle Stunden einberufen (Wintermeyer 2018). Im Vergleich zu den anderen Oppositionsparteien sticht sie damit nicht als übermäßig aktive Kraft heraus. Nach wie vor zielt die inhaltliche Fokussierung auf die Kernthemen Migration, Integration, Asyl, Innere Sicherheit und Ordnung. Wir vertreten daher die These, dass die AfD die jeweiligen Instrumente im Sinne einer fundamentaloppositionellen Politik einsetzt, die darauf abzielt, die Öffentlichkeit und vor allem eigene Anhänger_innen für das spezifische Kritikprofil der AfD zu sensibilisieren. Man kann auch von einer „Erpressungspartei“ sprechen, die populistisch die anderen Parteien vor sich hertreiben will.

Das Kerngeschäft der AfD sind gezielte Konfrontationen, die durch die intelligente Nutzung von Social Media verstärkt werden. Mit zugespitzten Aussagen, die häufig die anderen Parteien düpieren sollen, unterstreicht sie, dass für sie die Praxis der Polarisierung ein wesentliches Instrument der politischen Auseinandersetzung ist. Polarisiert wird teilweise nur, um Debatten für die Sozialen Netzwerke zu produzieren. Mit ihrer Skandalisierungs- und Provokationsstrategie gelingt es der AfD, die Mechanismen der Aufmerksamkeitsökonomie professionell zu nutzen. Bislang gelang es der AfD jedoch in kaum einem Parlament, andere Fraktionen für ihre Ideen zu gewinnen oder den professionellen Parlamentsbetrieb nachhaltig zu stören. Nur in SachsenAnhalt konnte sie einige Stimmen aus dem Lager der regierenden CDU gewinnen, um einen Untersuchungsausschuss gegen Linksextremismus durchzusetzen.

Während die Wählerschaft der AfD zu einem nicht unerheblichen Teil aus der Arbeiter_innenschaft kommt, liegt der Anteil der ehemaligen Arbeiter_innen in der AfD-Bundestagsfraktion bei null Prozent. Dagegen liegt der Anteil an Akademiker_innen bei $44 \%$. Kurz hinter den Grünen haben sie mit knapp $22 \%$ auch die zweithöchste Promovend_innenquote im Deutschen Bundestag. Im Kampf gegen das Establishment hat sich in der AfD eine eloquente und kommunikationsstarke Elite herausgebildet, die von Journalist_innen, Rechtsanwält_innen, Wissenschaftler_innen und Selbstständigen getragen wird.

Beobachter wie Bernd Gäbler gehen sogar so weit, von einer „Partei von Journalisten “ zu sprechen (Gäbler 2017, S. 13). Neben den Journalist_innen sind es die ausgebildeten Jurist_innen, die für die AfD nicht nur quantitativ wichtig sind, sondern auch, um eigenwillige Rechtsinterpretationen zu entwickeln und zu kommu- 
nizieren. Sie tragen außerdem dazu bei, das Image einer Law-and-Order-Partei zu bedienen. In den Reihen der AfD-Bundestagsfraktion befinden sich vier verbeamtete Richter und Staatsanwälte sowie sechs freiberufliche Richter und Notare - das sind knapp elf Prozent aller Abgeordnetenmandate der AfD. Zum Law-and-OrderBereich gehören auch die Polizist_innen und Soldat_innen, auf die bereits hingewiesen wurde. Laut AfD sollen sich unter den gegenwärtig rund 35.000 Mitgliedern etwa 2100 Berufssoldat_innen befinden. Zur AfD-Bundestagsfraktion gehörten 2019 elf frühere Berufs- und Zeitsoldat_innen.

Unter den AfD-Mandatsträger_innen finden sich zudem überproportional viele Selbstständige und freiberuflich Tätige. Zusammengenommen liegt ihr Anteil bei etwas mehr als $41 \%$ aller AfD-Bundestagsabgeordneten. Rund die Hälfte der AfD-Parlamentarier_innen war zu einem früheren Zeitpunkt bereits Mitglied in (mindestens) einer anderen Partei (Rütters 2019). In der Literatur ist der Standpunkt verbreitet, die AfD ziehe diejenigen an, die von der christdemokratischen Regierungspolitik unter Merkel enttäuscht seien und die sich ein entschieden konservativeres politisches Profil wünschten. Wobei sich diese Enttäuschung keineswegs alleine auf inhaltliche Positionen beziehe, sondern vielfach auch darin begründet liege, dass die Enttäuschten in ihren ehemaligen Parteien kaum Karriereperspektiven hatten. Diese Argumentation stützen einige Fakten aus unserer Untersuchung (vgl. Schroeder et al. 2017): Annähernd die Hälfte $(46,3 \%)$ der zuvor einer anderen Partei angehörenden AfD-Abgeordneten entfallen auf die CDU. An zweiter Stelle liegt die FDP (12,2 \%).

\section{Fazit}

Das Rätsel der AfD besteht darin, dass sie die erfolgreichste neu gegründete Partei im Hinblick auf schnelle und nachhaltige Beeinflussung der öffentlichen Debatten und Wahlerfolge ist, obwohl es über Jahrzehnte hinweg für unwahrscheinlich gehalten wurde, dass sich eine rechtspopulistische Partei dauerhaft im deutschen Parteiensystem verankern könne. Der Erfolg der AfD ist kein Selbstläufer. Schon die heterogene Koalition von bewegungs- und parlamentsorientierten Kräften mit ihren zuweilen widersprüchlichen Haltungen und Zielen bringt diese Partei ein ums andere Mal in existenzielle Krisen. Weiterhin offen ist insofern, ob es die AfD schafft, die Breite ihrer Wähler_innenbasis zu erhalten oder ob sie in den Fängen des Rechtsextremismus erstickt und damit dem „Schicksal“ aller anderen Rechtsaußenparteien in Deutschland folgt.
Funding. Open Access funding provided by Projekt DEAL.

Open Access. Dieser Artikel wird unter der Creative Commons Namensnennung 4.0 International Lizenz veröffentlicht, welche die Nutzung, Vervielfältigung, Bearbeitung, Verbreitung und Wiedergabe in jeglichem Medium und Format erlaubt, sofern Sie den/die ursprünglichen Autor(en) und die Quelle ordnungsgemäß nennen, einen Link zur Creative Commons Lizenz beifügen und angeben, $\mathrm{ob}$ Änderungen vorgenommen wurden.

Die in diesem Artikel enthaltenen Bilder und sonstiges Drittmaterial unterliegen ebenfalls der genannten Creative Commons Lizenz, sofern sich aus der Abbildungslegende nichts anderes ergibt. Sofern das betreffende Material nicht unter der genannten Creative Commons Lizenz steht und die betreffende Handlung nicht nach gesetzlichen Vorschriften erlaubt ist, ist für die oben aufgeführten Weiterverwendungen des Materials die Einwilligung des jeweiligen Rechteinhabers einzuholen.

Weitere Details zur Lizenz entnehmen Sie bitte der Lizenzinformation auf http://creativecommons.org/licenses/by/4.0/deed.de.

\section{Literatur}

Decker, F. (2016). Die „Alternative für Deutschland“ aus der vergleichenden Sicht der Parteienforschung. In A. Häusler (Hrsg.), Die Alternative für Deutschland (S. 7-23). Wiesbaden: Springer.

Gäbler, B. (2017). AfD und Medien. Analyse und Handreichungen. OBSArbeitsheft 92. Frankfurt a. M.: Otto Brenner Stiftung.

Höcke, Björn (2019). Zukunft der AfD. In: Deutschland-Kurier, 4.-6. Febr. 2019.

Rütters, P. (2019). Zum Sozialprofil der AfD-Abgeordneten im Bundestag: bedingt parlamentsfähig. Zeitschrift für Parlamentsfragen, 50, 59-80.

Schroeder, W., \& Weßels, B. (Hrsg.). (2019). Rechtspopulistische Landnahme in der Öffentlichkeit, im Elektorat und in den Parlamenten. In. Dies. (Hrsg.),Smarte Spalter (S. 9-43). Bonn: Dietz.

Schroeder, W., Weßels, B., Neusser, C., \& Berzel, A. (2017). Parlamentarische Praxis der AfD in deutschen Landesparlamenten. WZB Discussion Paper. Berlin: Wissenschaftszentrum Berlin für Sozialforschung (WZB).

Wintermeyer, F. (2018). Neubeginn in der parlamentarischen Praxis: Eine Untersuchung am Beispiel der Alternative für Deutschland (AfD). Masterarbeit FU-Berlin. 